\section{Bringing together population and quantitative genetics}

\author{
A Primer of Ecological Genetics \\ JK Conner and DL Hartl \\ Sinauer Associates Inc., Massachusetts, USA; 2004. 304pp. \\ \$34.95, paperback. ISBN 087893202X. \\ Heredity (2005) 95, 179-180. doi:10.1038/sj.hdy.6800684 \\ Reviewed by JM Cano Arias
}

Natural selection acts on the phenotype, although evolutionary change is only possible through changes to the genotype. Despite that fact, the genetic basis of phenotypic evolution has traditionally been studied by two complementary, but often disconnected, approaches. On the one hand, population genetics studies the genotypic variation between and within species and/or populations, but, except for a few model organisms, the genetic markers don't provide information about phenotypic variation. On the other hand, quantitative genetics infers the genetic architecture of quantitative traits treating the underlying coding genes as a black box. Recent theoretical and technical developments in population and quantitative genetics, such as QTL mapping, or the use of molecular markers to estimate pedigrees, allowed the development of Ecological Genetics as a new discipline on its own.

Until now, a textbook aimed at undergraduates and early postgraduates, which integrates both population and quantitative genetics, has been lacking. In six chapters, Conner and Hartl have successfully filled this gap; two chapters about population genetics and two about quantitative genetics provide the basis to address the study of natural selection and conservation of natural populations in the last two chapters.

By exposing the way gene frequencies should behave according to the assumptions of the Hardy-Weinberg $(\mathrm{H}-\mathrm{W})$ model, the authors examine how such different processes as mutation, nonrandom mating, migration, genetic drift and selection can lead to departures from the expected equilibrium. This sensible approach will not panic population geneticists, as usually happens when the gene frequencies of the loci under study depart from the $\mathrm{H}-\mathrm{W}$ equilibrium. Rather, it aids understanding of the likely causes behind the observed genotypic variation (ie gene flow, inbreeding, etc). Conner and Hartl start by relaxing one $\mathrm{H}-\mathrm{W}$ assumption at a time and then examine the effect of two or three evolutionary forces acting together. By doing this, they accomplish accessible explanations of complex concepts such as Wright's shifting balance theory and the adaptive landscape.

The quantitative genetics section combines the information about changes in allele frequencies from previous chapters with the explanation of the additive and dominant types of gene action. It follows the notions of breeding value, heritability, additive and dominance variances and the different ways to estimate these parameters (ie offspring-parent regression, full-sib and half-sib breeding designs). The distinction between phenotypic plasticity and local adaptation is addressed from a reaction norm perspective. Additionally, the comparison of differentiation indexes of quantitative traits $\left(Q_{\mathrm{ST}}\right)$ and neutral genetic markers $\left(F_{\mathrm{ST}}\right)$ is advocated to better understand the relative importance of natural selection and genetic drift in population differentiation.

The book explicitly considers the multivariate nature of phenotypic evolution by introducing the concepts of genetic and environmental covariances and correlations, as well as their importance in facilitating or constraining phenotypic evolution. The possible causes of genetic covariation are presented, giving special attention to pleiotropy and linkage disequilibrium. That way, the reader is already prepared to understand the rationale of QTL mapping, which is presented as one of the most promising approaches for uncovering the genetic mechanisms underlying phenotypic variation.

Artificial selection on single traits is discussed as a way of directly measuring the capacity of the genotypes to respond to selection, and allows the authors to introduce the breeder's equation to measure the response to selection. However, since selection acts on several traits at a time in the wild, Conner and Hartl propose the so-called Chicago approach to study the effect of natural selection on phenotypes. From that perspective, several definitions of fitness and its components are provided. The text introduces the concept of the fitness function and how it is estimated, and, based on the different shapes of the fitness function, it explains the basic types of phenotypic selection (ie directional, stabilizing and disruptive or, more appropriately, linear and nonlinear responses). In turn, the book extends the previous concepts to its multivariate equivalents (ie correlational selection and fitness surface) and shows how the selection gradients can be combined with multivariate quantitative genetic estimates (the G-matrix) to predict short-term evolutionary outcomes.

The last chapter covers applied ecological genetics, providing a quick overview of topics such as conservation of evolutionary significant units, extinction risk, invasive species and the impact of genetic-modified organisms.

All in all, this is a very well written book that should serve as a basic reference for future courses in ecological genetics. Although a more extensive treatment of the identity-by-descent concept and its relevance to the estimation of population and quantitative genetic parameters would have been welcomed, as well as a brief introduction to microarray analyses as a way to measure gene expression.

Throughout the book, the bibliographic references in the text are kept to a minimum, making the reading easy. Maths are avoided, presenting only the relevant formulae and models for each chapter. Every chapter has worked examples, problems and proposed readings with related questions. Another strength of this book is the extremely informative graphs illustrating the many different concepts. The well-planned structure, easy reading style and extensive coverage make this book valuable not only to undergraduate students but also for 
population and quantitative geneticists aiming to expand their scope of their own research.

JM Cano Arias

Ecological Genetics Research Unit, Department of Bio- and Environmental Sciences,
PO Box 65 (Biokeskus 3, Viikinkaari 1), University of Helsinki, Helsinki FIN-00014, Finland

E-mail: jose.canoarias@helsinki.fi 